\title{
Power Window Anti-Pinch Control Based on Hall Pulse Signal and Mechanical Power
}

\author{
Xiwen Tang, Li Jiang, Wei Han, Qiwei Gao \\ School of Radar Confrontation, National University of Defense, Hefei, China \\ shelley7983@163.com,jili1130@163.com,37828843@qq.com,183767243@qq.com
}

Keywords: automotive power window anti-pinch; hall pulse signal; mechanical power; the anti-pinch region

Abstract. In order to avoid the erroneous judgement produced by the motor discontinuous current, which is caused by the gap between mechanical friction and mechanical transmission. the power window anti-pinch system model is set up based on the MATLAB/Simulink, and the simulation has been done. The simulation results show that: The power window motor hall pulse signal detection and mechanical power calculation combining power window anti pinch control strategy can accurately detect the anti-pinch region, effectively to avoid inadvertent occlusion accidents, enhance the safety of the car.

\section{Introduction}

Power windows have been popularized in modern vehicles to improve the convenience of vehicle operation, avoid driver distractions and improves the safety of vehicle. However, there are also some dangers in the power window. When the window is rising, passengers may be pinched, which may endanger the safety of the body and life. In order to ensure the safety of the passengers, it is necessary to study the power window anti-pinch control technology[1-3].

At present, the anti-pinch control of automobile power windows basically adopts the method of detecting the motor current to judge whether the car window meets an obstacle[2]. As the window mechanical friction changes or the gap of mechanical transmission will cause the motor current discontinuity resulting in control system misjudgment[4-7]. In this paper, a power window anti-pinch control strategy combined with the detection of motor Hall pulse signal and the calculation of motor mechanical power is used to improve the accuracy of obstacle detection.

\section{Circuitry and mechanical equations for window motors}

$$
\begin{gathered}
U(t)=E(t)+R I(t)+L \frac{d I}{d t} \\
E(t)=K_{e} \Omega(t) \\
T_{e}=K_{T} I(t) \\
J \frac{d \Omega}{d t}=T_{e}-B \Omega(t)
\end{gathered}
$$

In the above formula:

$$
\begin{aligned}
& U(t) \text {-Armature Voltage }(V) ; E(t) \text {-Back electromotive force }(\mathrm{EMF})(V) ; \\
& I(t) \text {-Armature current }(A) ; R \text {-Armature internal resistance }(\Omega) ; \\
& L \text {-Armature inductor }(H) ; T_{e} \text {-Electromagnetic torque }(N \cdot m) ;
\end{aligned}
$$


$J$-Moment of inertia $\left(N \cdot \mathrm{m} /\left(\mathrm{rad} \cdot \mathrm{s}^{-2}\right)\right) ; B$-Viscous Frictional Coefficient $\left(N \cdot \mathrm{m} /\left(\mathrm{rad} \cdot \mathrm{s}^{-2}\right)\right)$;

$\Omega$-Motor speed $(\mathrm{rad} / \mathrm{s}) ; K_{e}-\mathrm{EMF}$ Constant $\left(\mathrm{V} /\left(\mathrm{rad} \cdot \mathrm{s}^{-1}\right)\right)$;

$K_{T}$-Torque Constant $(N \cdot m / A)$.

\section{Laplasse transform expression}

$$
\begin{aligned}
U(s) & =E(s)+R I(s)+L s I(s) & & \text { Eq. } 5 \\
E(s) & =K_{e} \Omega(s) & & \text { Eq. } 6 \\
T_{e} & =K_{T} \Omega(s) & & \text { Eq. } 7 \\
J S \Omega(s) & =T_{e} B \Omega(s) & & \text { Eq. } 8
\end{aligned}
$$

\section{Window motor mechanical output}

$$
\begin{gathered}
T_{e}=T_{L}+T_{0} \\
T_{L}=T_{1}+B \Omega(t) \\
T_{1}=\frac{r}{n}\left(M \frac{d^{\prime \prime} D}{d t^{\prime \prime}}+F_{v} \frac{d D}{d t}+F_{s}+M g\right)
\end{gathered}
$$

By Laplasse transform, we can get:

$$
\begin{aligned}
T_{L} & =T_{1}+B \Omega(s) \\
D & =\frac{T_{1} \frac{n}{r}-F_{s}-M g}{M s^{2}+F_{v} s}
\end{aligned}
$$

In the above formula:

$T_{L}$-Load Torque $(N \cdot m) ; T_{1}$ - The component independent of the speed in the load torque $(N \cdot m)$;

$r$-Winding Wheel Radius $(m) ; n$-Mechanical Transmission Ratio ;

$M$-Window Weight $(\mathrm{kg}) ; F_{v}$-Sliding Friction Coefficient $\left(N /\left(m \cdot s^{-1}\right)\right)$;

$F_{s}$-Static Friction $(N)$; D-Window Position $(m) ; \mathrm{g}-$ Gravitational Acceleration $\left(9.81 \mathrm{~m} / \mathrm{s}^{2}\right)$.

The above DC motor armature circuit equations and mechanical power circuit equations are combined, and the following equation can be obtained.

$$
D=\frac{K_{T} \frac{U-E}{L s+R}-T_{0}-B \Omega-F_{s}-M g}{M s^{2}+F_{v} s}
$$

Eq. 14

\section{Power window anti-pinch control considering mechanical power calculation}

First, the motor speed is calculated based on the position calibration reference value of the power window obtained by the above method and the collected Hall pulse signal, and compare with the position calibration reference value of the power window to determine the specific position where the power window is located. According to European 74/60 / EEC and the United States FMVSS118 regulations related standards to determine whether the window in the anti-pinch area or 
the top of the window[4,5][10].

Secondly, in this paper, in order to avoid the system misjudging the following situation as obstacle encountered: the motor current surge at the initial stage of the electric car window, the change of the electric window friction (static or dynamic) and the motor current interruption caused by the mechanical transmission gap, the method of mechanical power calculation is used instead of motor current detection method to prevent erroneous judgement and accurately identify whether obstacles are encountered in the process of lifting windows. The mechanical power calculation adopts the method of product of current and angular velocity to ensure that the calculation result can avoid the interruption of the motor current due to the friction change (static or dynamic) of the window or the transmission gap of the mechanical system so as to improve the accuracy of the judgment of the obstacle and effectively avoid the error anti-pinch situation occurred.

\section{Simulation and simulation results analysis}

Based on the above analysis of permanent magnet DC motor model and the detection principle of power window anti-pinch area, a simulation model of automotive power window anti-pinch system is built by using MATLAB/Simulink. The model is shown in Figure 1 and simulation results are shown in Figure 2 and Figure 3.

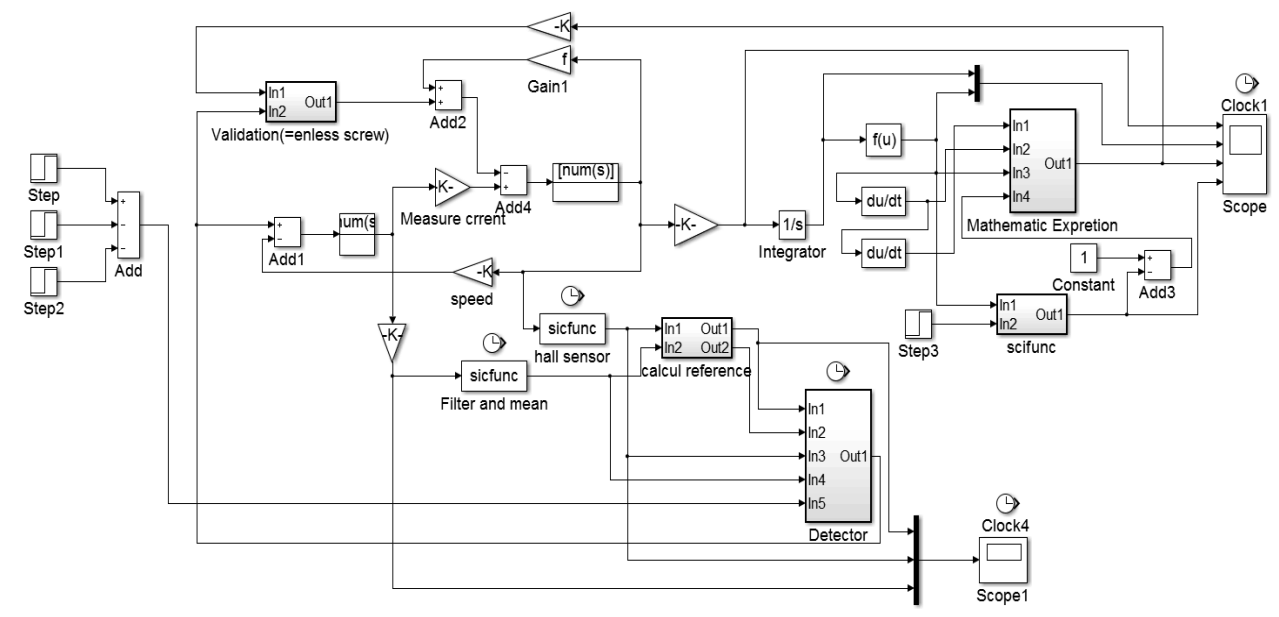

Fig.1 Simulation model of anti-pinch system for automobile power window
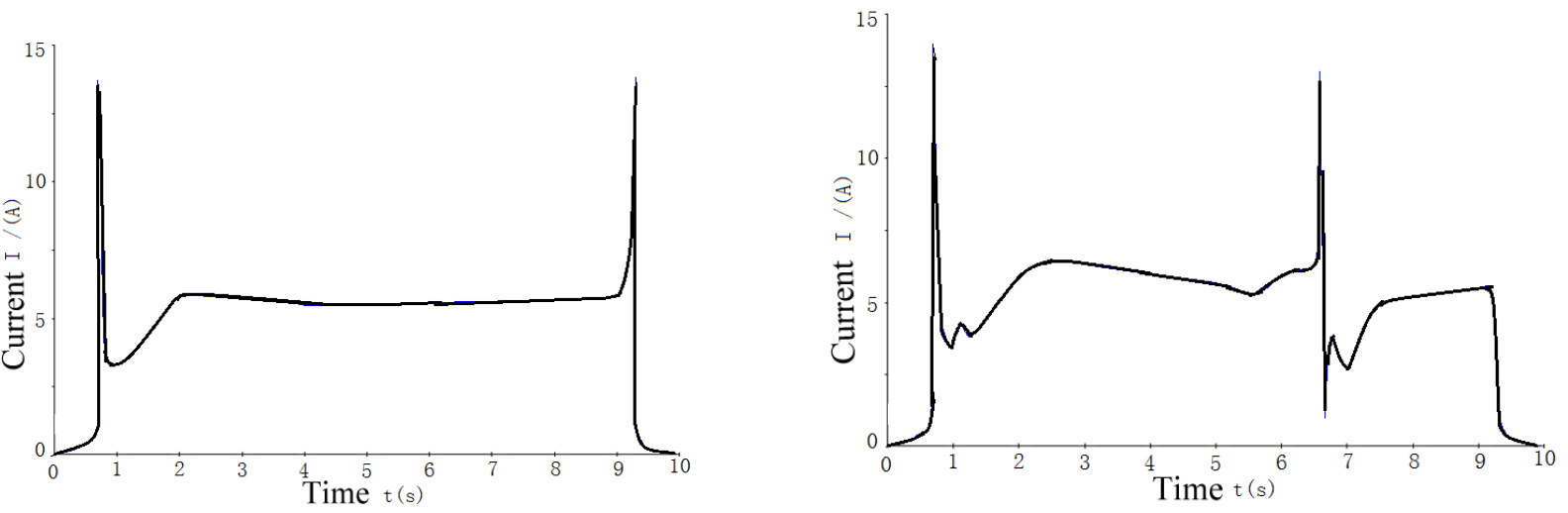

Fig. 2 Motor armature current waveform

Fig.3Motor armature current waveform when encountering obstacle

The simulation results show that the automatic anti-pinch function of the power window can be realized by comprehensively considering the detection of the motor Hall pulse signal and the calculation of the mechanical power. 


\section{Conclusion}

The automobile power window anti-pinch control strategy is combined with motor Hall pulse signal detection and mechanical power calculation, which can accurately determine the specific location of the power window. This control strategy also can effectively avoid the misjudgement of obstacles encountered because of the interruption of motor current caused by the surge of current in the initial stage of the power window, the change of the friction (static or dynamic) of the power window and the mechanical transmission gap, improve the accuracy of judgments on obstacles and improve the safety of vehicles.

\section{References}

[1] Yanhai Du. The design of anti-pinch window lifter based on double hall sensor[D]. Donghua University, 2014

[2] Weize Ma and Dedong Meng. The Discussion about Anti-pinch Technology for Automotive Electric Window[J]. Tianjin Auto, 2008 (10): 44-46.

[3] Chaohui Fu. Discussion on Anti-pinch Technology for Power Window[J]. Auto Electric Parts, 2012 (7): 15-18.

[4] Chenchen Ai. Design of automobile power window anti-pinch control System based on $\mu \mathrm{C} / \mathrm{OS}$ II[D]. Hefei University of Technology, 2013.

[5] Weize Ma, Shenke Zhang and Hongjie Wang. The Design of Anti-pinch Car Windows Using Hall Sensor[J]. Automotive engineering, 2008, 30(12): 1122-1123.

[6] Guojun Dai, Xiang Zhang and Huaixiang Zhang. Modeling and Implementation of Power Window Anti-pinch System[J]. Automotive engineering, 2008:30(6):539-541.

[7] Shizhong Xu. Design of the Power Windows Anti-pinch Based on the Current Ripple Waveform Transformation with Pure Hardware[J]. Journal of Lujiang University, 2014, 22(1): 11-16.

[8] Youhua xie. Research on CAN/LIN based lightning and power window control[D]. Shanghai Jiao Tong University, 2007.

[9] Yong Tian and Ying Fang. On the Anti-pinch Design of Automotive Power Window[J]. Auto Electric Parts, 2014 (8): 12-15.

[10]Haiyan $\mathrm{Wu}$, Zhihong $\mathrm{Wu}$, and Yuan Zhu et al. Design window lifter of door control modules[J]. Electronic Products, 2006 (9): 74-77. 